\title{
REFLEXIONES SOBRE NUESTRAS PRÁCTICAS. UN ACERCAMIENTO A LA MODALIDAD DE INTERVENCIÓN EN DISPOSITIVOS LABORALES DE SALUD MENTAL EN EL SECTOR PÚBLICO DE LA CIUDAD DE BUENOS AIRES
}

\author{
REFLECTIONS ON OUR PRACTICES. A RAPPROCHEMENT TO \\ THE MODALITY OF INTERVENTION IN LABOR PROGRAMS \\ IN MENTAL HEALTH IN THE PUBLIC SECTOR OF THE CITY OF \\ BUENOS AIRES
}

\section{Daniela Testa ${ }^{1}$}

\section{Resumen}

Introducción: Este trabajo tiene el propósito de conocer la modalidad de intervención del terapista ocupacional en dispositivos laborales de salud mental para adultos, pertenecientes al sector público de salud.

Método: Es una investigación de tipo cualitativo exploratorio desarrollada en la Ciudad de Buenos Aires (Argentina), durante el año 2006. Los datos se obtuvieron de documentación, y a través de entrevistas a los terapistas ocupacionales que conformaron la muestra.

Los resultados obtenidos significan la descripción y sistematización de las prácticas del terapista ocupacional en este campo, y demuestran la importancia de la Terapia Ocupacional a la hora de generar cambios en las formas concretas de atención sanitaria.

Conclusión: Aunque se estableció una modalidad de intervención particularizada en cada tipo de dispositivo, según la vinculación entre las intervenciones más frecuentes, los objetivos de las mismas y las metas que se propone cada dispositivo, se identificaron también rasgos en común en dichas modalidades. Tales similitudes se expresaron a través de la prevalencia de las intervenciones basadas en el propósito de favorecer la integración social a través de la creación de nuevos espacios sociales de inclusión, y en el modo de concebir el problema de la salud mental asumiendo la complejidad del ser humano en sus múltiples dimensiones.

Licenciada en Terapia Ocupacional. Maestranda en Diseño y Gestión de Programas y Políticas Sociales (FLACSO. Argentina), con tesis en elaboración. Servicio de Terapia Ocupacional del Hospital Interdisciplinario Psicoasistencial José T. Borda. Buenos Aires. Argentina. Correo postal: Portela 708- Ciudad Autónoma de Buenos Aires. Argentina. CP 1406. Teléfono: 0054-11 4612 - 1791 Email: danitestu@yahoo.com.ar 


\section{Palabras clave}

Modalidad de intervención, dispositivo laboral, salud mental.

\section{Summary}

Introduction: This work has the intention of knowing the modality of intervention of the occupational therapist in labour programs of mental health for adults, belonging to the public sector of health.

Method: It is an exploratory investigation of qualitative type developed in the City of Buenos Aires (Argentina) during the year 2006.

The information was obtained of documentation, and across interviews realized to the occupational therapists who shaped the sample.

The obtained results mean a description and systematizing of the practices of the occupational therapist in this field, and demonstrate the importance of Occupational Therapy to generate changes in the specific forms of health care.

Conclusion: Though there was established a modality of intervention distinguished in every type of program, according to the relation of the most frequent interventions, his aims and the goals that every program proposes, also some similar characteristics could be identified.

Such similarities expressed across the prevalence of the interventions based on the intention of favoring the social integration across the creation of new social spaces of inclusion and also in the way of conceiving the problem of the mental health assuming the complexity of the human being in his multiple dimensions.

\section{Keywords}

Modality of intervention, labour program, mental health.

\section{INTRODUCCIÓN}

En Terapia Ocupacional el trabajo ha sido utilizado tanto como fin o medio terapéutico, con distintos propósitos (adaptativo-normalizador, moralizador, de re-inserción social, etc.) de acuerdo con los diferentes marcos socio- históricos, ideológicos y filosóficos. Del mismo modo las actividades laborales han estado presentes en el desarrollo de las conceptualizaciones y de las prácticas de la Terapia Ocupacional.

Numerosas publicaciones y comunicaciones presentadas en Jornadas, Congresos y otros encuentros científicos de Terapia Ocupacional reflejan que la temática de "lo laboral" ha sido una preocupación presente en las prácticas de los terapistas ocupacionales.

Dan cuenta de ello, la existencia de dispositivos laborales con diferentes modos de organización, tales como talleres ocupacionales, micro emprendimientos cooperativos, empresas sociales, etc., instrumentados con el objetivo de disminuir la situación de desventaja y vulnerabilidad psicosocial, y mejorar la situación laboral y de inserción relacional de la población asistida. Esta diversidad se refleja también en la población objetivo a la que están dirigidos: adolescentes, ancianos, (1) personas con trastorno mental severo, personas que portan discapacidades mentales y motoras. (2)

Datos surgidos del relevamiento previo a la presente investigación en las instituciones efectoras de la red de salud mental del Gobierno de la Ciudad de Buenos Aires, revelaron la existencia de cinco tipos de dispositivos laborales para adultos, cuatro de ellos surgidos y creados por terapistas ocupacionales durante la última década. ¿Cuáles son y qué modalidades de abordaje proponen cada uno de ellos? ¿Cómo es concebido el 
trabajo en relación a las intervenciones terapéuticas instrumentadas? Los "nuevos dispositivos" creados, todos ellos en hospitales psiquiátricos, ¿implican alguna transformación en las prácticas de los terapistas ocupacionales en este campo?

Son éstas algunas de las preguntas que motivaron este estudio, que tiene el propósito de describir la modalidad de intervención de los terapistas ocupacionales en dispositivos laborales para adultos en Salud Mental en el ámbito del sub-sector de salud pública de la Ciudad de Buenos Aires, basado en el supuesto que conocer acerca de los procedimientos, técnicas y modalidades, devela la importancia y potencial transformador de las prácticas concretas de la Terapia Ocupacional en los contextos institucionales en que se instrumentan. (3)

\section{Marco Conceptual}

\section{Breves Consideraciones Teóricas}

Para la construcción del objeto de estudio de esta investigación, la modalidad de intervención del terapista ocupacional en dispositivos laborales en salud mental, he tomado en cuenta las siguientes variables principales: a) Evaluación b) Asesoramiento y orientación c) Entrenamiento y d) Objetivo general de las intervenciones.

Estas variables relacionadas entre sí posibilitan conocer acerca de los modos de abordar la problemática de salud mental propuestas desde los dispositivos laborales, porque se considera que el relevamiento de los datos a obtener a través de ellas, contienen los conceptos y nociones que caracterizan las intervenciones terapéuticas de los terapistas ocupacionales dentro de un contexto determinado.

Las variables contextuales, a) Características del Dispositivo Laboral b) Localización c) Población objetivo y d) Actividades de enlace, fueron seleccionaron como tales por su importancia en relación con las variables principales. El papel de estas variables contextuales puede ser facilitador directo o no, de las intervenciones y las modalidades a ser desarrolladas por los terapistas ocupacionales.

Describen algunas características de organización y funcionamiento de los dispositivos laborales, datos sobre la población que participa y / o asiste a los mismos, y cómo se relaciona cada dispositivo con otros actores de la comunidad, ya sea dentro de la institución de referencia o por fuera de la misma.

El desarrollo teórico de Liliana Paganizzi (4) y de Maria Rita Martínez Antón, (5) específicos de la Terapia Ocupacional en la Argentina, resultó de especial importancia para abordar el análisis de la modalidad de las intervenciones. El planteo de José Schiapa Pietra (6) y de Benedetto Saraceno (7) arrojó luces sobre las variables contextuales y su relación con las diversas formas de pensar las intervenciones y su relación con lo micro social y los procesos sociales más amplios.

Antes de continuar, es clave explicitar como entenderé los siguientes conceptos principales del estudio:

Modalidad de intervención: se refiere al modo en que el terapista ocupacional trata con aquello que lo convoca, se refiere al cómo desarrolla y/o aplica en la práctica el conjunto de técnicas y métodos instrumentados con el propósito de lograr un objetivo determinado.

Intervención: implica tomar parte en un asunto, mediar, interponerse. Se utiliza este término para designar en general las acciones del terapista, incluyendo acciones referidas no sólo a lo que se entiende habitualmente como el campo de la salud, sino también en el campo comunitario, laboral, etc. Por lo tanto se hace referencia a la aplicación de técnicas y métodos implementados en el ejercicio de su práctica profesional.

Dispositivo laboral en salud mental: se refiere a un contexto, con fines terapéuticos (delimitado en un espacio y tiempo determinados) organizado a partir del eje actividad laboral, que se desarrolla y está dirigido específicamente a personas con padecimiento psíquico. Se considerarán dispositivos de distinta complejidad en su organización, tanto el que constituye una institución en si mismo, como los que se incluyen en otras instituciones, como programas, proyectos, etc. siempre y cuando tengan alguna inscripción institucional. 


\section{MÉTODO}

Se realizó una sistematización en base a las prácticas desarrolladas por terapistas ocupacionales en dispositivos laborales en el campo de la salud mental.

Dicha investigación se enmarcó en cuanto a su diseño y desarrollo, dentro de un enfoque de tipo cualitativo exploratorio.

Las fuentes de datos: se obtuvieron de la lectura de documentación referida al tema, tratándose en su mayoría de textos inéditos o mimeografiados, comunicaciones en eventos científicos, y a través del relevamiento de información proporcionada individualmente a través de entrevistas, realizadas a los terapistas ocupacionales que conformaron la muestra.

La muestra: está conformada por las modalidades de intervención de veintiún terapistas ocupacionales, que se desempeñan en distintos dispositivos laborales de los hospitales psiquiátricos de la ciudad (Hospital José T. Borda y Hospital Braulio Moyano) y Talleres Protegidos de Rehabilitación Psiquiátrica.

La población en estudio estuvo constituida por los terapistas ocupacionales que se desempeñan en dispositivos laborales para adultos en el ámbito de la salud mental; un total de veintisiete terapistas ocupacionales de la Red de Salud Mental del Gobierno de la Ciudad de Buenos Aires, dedican su carga horaria (en parte o en su totalidad) a desempeñarse profesionalmente en dispositivos laborales para adultos.

La estrategia metodológica se realizó en tres etapas, a saber:

a- Etapa exploratoria: se llevó adelante el relevamiento institucional y la construcción de los instrumentos de trabajo a través de la planificación de la entrevista y del criterio de observación a tenerse en cuenta durante las mismas.

La planificación de la entrevista consistió en la elaboración de una guía para la recolección de datos en función de las variables planteadas, que focalizaron la entrevista en cuestiones precisas y pertinentes. Se formularon preguntas abiertas, debido al interés de recabar predominantemente datos cualitativos, aunque también se realizaron algunas preguntas cerradas referidas específicamente a relevar datos o características del contexto. (8)

b- Trabajo de campo: los componentes utilizados para el mismo fueron la aplicación de las técnicas de Entrevista. La modalidad utilizada fue la de entrevista semi-estructurada, la cual permite una mayor libertad a la iniciativa de expresión del entrevistado, por tratarse en su mayoría de preguntas abiertas que se realizan cara a cara dentro de una conversación. Ofrece también mayor flexibilidad al entrevistador para adaptarse a las diferentes circunstancias concretas que caracterizan al trabajo de campo.

Las entrevistas se efectuaron en su totalidad en el lugar de trabajo estudiado y la duración aproximada de las mismas fue de 60 minutos. Todas fueron grabadas, previo acuerdo con el entrevistado.

Se tomo en cuenta dos aspectos considerados como "limitaciones inherentes a la técnica de entrevista en sí misma", que me llevaron a tomar algunos recaudos para el desempeño como entrevistadora. $(8,9)$

Uno de ellos, referido a "las limitaciones de la expresión verbal", invita a poner esmero en el uso correcto de la expresión oral y de la terminología específica, evitando de ese modo la utilización de términos que reflejen valoración por parte del entrevistador, generando un clima agradable y distendido para la conversación.

El otro, presenta la falta de "secreto y total anonimato" de las respuestas, como una limitación inevitable de este estudio, debido a las características propias de nuestra comunidad profesional local. Dado que es frecuente el conocimiento personal entre los profesionales y las instituciones en las que trabajan, sería posible inferir sobre lo participantes en el estudio. Para atenuar esta situación, se dio importancia a brindar información clara y precisa sobre el tipo de estudio que se realizaría, en el cual no se contemplaría la intención de realizar evaluaciones de procesos personales.

En relación al aspecto ético, previamente a la recolección de datos se dio a conocer a cada terapista ocupacional entrevistada, el objetivo y alcance del proyecto, respetándose el deseo de participar o no del mismo. 
c- Tratamiento de los Datos: Se utilizó la técnica de Análisis Temático, la cual consiste en "descubrir los núcleos de sentido que componen una comunicación cuya presencia o frecuencia signifiquen alguna cosa para el objetivo analítico trazado", orientado a detectar las unidades de significación que definen el discurso, ya que cualitativamente la presencia de determinados temas denotan valores y modelos de referencia presentes. (9) Se desarrolló en tres pasos, el del pre-análisis, en el cual se determinó la forma de categorización y los conceptos teóricos más generales que orientaron el análisis. Luego, se realizó la explotación del material, donde se elaboró la Matriz de Datos, seguida de la transformación de los datos obtenidos en bruto para llegar a la comprensión global del material. Por último el análisis final de los resultados, proponiendo inferencias y realizando interpretaciones previstas en el cuadro teórico.

\section{Resultados}

\section{Dispositivos Laborales}

Se identificaron cinco tipos diferentes de dispositivos laborales, a saber:

- Taller Protegido Terapéutico

- Taller Ocupacional

- Emprendimiento Social en Salud

- Programa Comunitario de Voluntariado

- Programa de Inserción Laboral

En relación con la creación y desarrollo histórico de los dispositivos estudiados, de los cinco tipos de Dispositivos Laborales, cuatro fueron creados en la última década por terapistas ocupacionales: el Taller Ocupacional, el Programa de Inserción Laboral, el Emprendimiento Social en Salud y el Programa Comunitario Solidario.

Considerando la historia de cada dispositivo laboral, es destacable la relación entre las condiciones sociohistóricas en las que surgieron y las características que ellos tomaron. La crisis y la desocupación de la década del 90 y sus repercusiones en las condiciones de exclusión y marginalidad, representaron un factor de influencia no sólo en la modificación de las prácticas terapéuticas, sino en la creación de los cuatro últimos dispositivos mencionados.

\section{Modalidad de Intervención}

1. Es posible establecer una modalidad de intervención particularizada en cada tipo de dispositivo laboral, según la relación que guardan las intervenciones terapéuticas que se realizan con mayor prevalencia, los objetivos generales de las mismas y las metas o misión que se propone cada dispositivo.

2. Se identificaron rasgos en común en las modalidades de intervención, en tres de esos cuatro dispositivos: el Emprendimiento Social en Salud, el Programa Comunitario Solidario y el Programa de Inserción Laboral. Ellos están dados por:

- la presencia de las intervenciones de autogestión, participación y solidaridad como prevalentes;

- y el objetivo general de favorecer la integración social a través de la creación de nuevos espacios de circulación, intercambios e inclusión social.

3. En relación con las intervenciones mencionadas, todas estas presentan la peculiaridad de operar en diferentes niveles de profundidad. La autogestión en el aspecto personal, de sostén del grupo y de capacidad de generar recursos para afianzar la estructura del dispositivo. La participación desde estar informado, opinar hasta modificar. La gestión administrativa, a nivel personal, institucional o intersectorial. El concepto de solidaridad, como intervención terapéutica, abarca una multiplicidad de significaciones y también distintos niveles de profundidad. En este caso, la noción de solidaridad identificada en las intervenciones, guarda relación especialmente con la mutualidad y la cooperación, alejándose de la noción de solidaridad emparentada con la caridad y el paternalismo. Se basa en la capacidad de modificar los contextos de vida a través de las acciones y actitudes de todos los días, desde el espacio de lo cotidiano. 
4. Las intervenciones de creatividad, orientación y asesoramiento fueron identificadas como relevantes en los distintos dispositivos, así como también las actividades de enlace entre distintas instituciones y actores sociales, por su capacidad de desdibujar los límites entre el adentro-afuera de las instituciones.

5. En todos los casos analizados, se manifestó la intención de reformular o transformar la práctica profesional, asumiendo el desafío de dar una respuesta viable a una problemática determinada, lo cual se vio reflejado en la modalidad de intervención instrumentada. Las intervenciones que se implementan en los dispositivos laborales, en mayor o menor medida, ejercen potencia sobre la lógica del asistido, intentando restablecer la lógica del ciudadano, tanto para el usuario como para el profesional de salud mental, ya que ambos intentan la creación y / o transformación a pequeña escala de un espacio social.

Es posible entonces, señalar una lógica de pensamiento presente en las distintas modalidades de intervención, que se refleja en el modo de analizar y concebir el problema de la salud mental, que se puede identificar con el Ilamado paradigma de la complejidad. (10) Dicho paradigma propone asumir la complejidad del ser humano como constitutiva y propia de su naturaleza e integrar las múltiples dimensiones que lo componen.

6. A continuación algunos conceptos que dan sustento a las modalidades de intervención analizadas: Los conceptos de salud, trabajo, rehabilitación psicosocial y vulnerabilidad psicosocial se piensan como las ideas fundamentales que dan marco a las intervenciones terapéuticas instrumentadas.

De este modo, y a partir de ellos, se rota el eje de la enfermedad mental hacia el de la situación de vulnerabilidad psico-social, donde se interviene ya no para reducir o controlar la enfermedad, sino para crear un contexto real de vida que produzca salud, solidaridad y creatividad. Se incluye la problemática de los derechos humanos y su incidencia en la situación social y de salud.

Otra de las ideas fundamentales es la de rehabilitación psicosocial, planteada como una práctica que trata al sufrimiento psíquico como la pérdida de la capacidad de generar sentido y con intermitencias entre los períodos de sufrimiento.

La Vulnerabilidad Psicosocial se propone como uno de los ejes de análisis e intervención, a partir del cual se ubica como sujeto vulnerable a aquél susceptible de ser dañado. A partir del aporte teórico realizado por Robert Castel se entiende a la vulnerabilidad psicosocial como el grado de fragilidad psíquica que la persona sufre por haber sido desatendida en sus necesidades básicas. (11)

En síntesis, en las distintas modalidades de intervención se revela la coexistencia de elementos propios de diferentes discursos (médico, filosófico, social, antropológico, jurídico, del poder, etc.), que combinados de variadas formas se traducen en las prácticas concretas; pudiéndose identificar como consecuencia, un recorrido de las mismas que va desde la complejidad hacia la especificidad de la Terapia Ocupacional en el campo de la salud mental, asumiendo los desafíos de las sociedades actuales y entendiendo la importancia de considerar aquellos elementos del contexto que inciden directamente en las estrategias de intervención implementadas.

Los Dispositivos Laborales, sus Objetivos Generales y las Intervenciones Terapéuticas que prevalecen. 


\begin{tabular}{|c|c|c|c|}
\hline \multicolumn{2}{|c|}{ Dispositivo Laboral } & Objetivo General & Intervenciones \\
\hline \multirow{2}{*}{$\begin{array}{c}\text { Taller Protegido } \\
\text { Terapéutico } \\
(*)\end{array}$} & $\begin{array}{c}\text { Talleres Protegidos } \\
\text { de Rehabilitación } \\
\text { Psiquiátrica } \\
\text { (Institución de la Red } \\
\text { de Salud Mental) }\end{array}$ & $\begin{array}{c}\text {-Mejorar la situación de salud } \\
\text { para lograr la inserción social del } \\
\text { paciente }\end{array}$ & $\begin{array}{l}\text { Implementación de variedad de } \\
\text { recursos terapéuticos, técnicas, } \\
\text { instrumentos y formas de abordaje } \\
\text { para la evaluación, planificación y } \\
\text { tratamiento. }\end{array}$ \\
\hline & $\begin{array}{c}\text { Taller Protegido y } \\
\text { Taller Pre-Laboral } \\
\text { (Dispositivos labora- } \\
\text { les que funcionan en } \\
\text { el Hospital Moyano) }\end{array}$ & $\begin{array}{c}\text {-Mejorar la situación de salud } \\
\text { para disminuir las desventajas y/o } \\
\text { secuelas de la enfermedad }\end{array}$ & $\begin{array}{c}\text { Evaluación psico-funcional } \\
\text { Entrenamiento } \\
\text { Asesoramiento } \\
\text { Gestiones internas }\end{array}$ \\
\hline \multicolumn{2}{|c|}{ Emprendimiento Social en Salud } & $\begin{array}{l}\text {-Transformar la institución psiquiá- } \\
\text { trica y los modos de asistencia. } \\
\text {-Crear espacios de intercambio e } \\
\text { integración social }\end{array}$ & $\begin{array}{l}\text { Autogestión } \\
\text { Solidaridad } \\
\text { Creatividad } \\
\text { Participación }\end{array}$ \\
\hline \multicolumn{2}{|c|}{ Taller Ocupacional } & $\begin{array}{l}\text {-Acompañar y guiar el proceso } \\
\text { de implicación del sujeto en la } \\
\text { situación laboral }\end{array}$ & $\begin{array}{c}\text { Orientación } \\
\text { Asesoramiento }\end{array}$ \\
\hline \multicolumn{2}{|c|}{ Programa Comunitario Solidario } & $\begin{array}{l}\text {-Lograr la inserción social del pa- } \\
\text { ciente a través de la realización } \\
\text { de actividades solidarias. } \\
\text {-Crear un espacio de partici- } \\
\text { pación para el ejercicio del rol } \\
\text { solidario }\end{array}$ & $\begin{array}{c}\text { Evaluación y Planificación } \\
\text { Autogestión } \\
\text { Participación } \\
\text { Solidaridad }\end{array}$ \\
\hline \multicolumn{2}{|c|}{ Programa de Inserción Laboral } & $\begin{array}{c}\text {-Crear espacios de integración } \\
\text { laboral }\end{array}$ & $\begin{array}{l}\text { Orientación / Asesoramiento } \\
\text { Autogestión / Participación }\end{array}$ \\
\hline
\end{tabular}

Fuente: elaboración propia basada en los datos de las entrevistas realizadas, 2006.

(*) El tipo de Dispositivo Laboral Taller Protegido Terapéutico, se localiza en la institución Talleres Protegidos de Rehabilitación Psiquiátrica (que forma parte de la Red de Salud Mental de la Ciudad) y también dentro de la institución Hospital Moyano, donde se suma a otros variados servicios de salud mental.

\section{DisCUSIÓN}

Se ha intentado en este estudio sistematizar las modalidades de intervención en dispositivos laborales de salud mental del sector público de la Ciudad de Buenos Aires, apuntando a reconocer aspectos relevantes y específicos de las mismas. A continuación, algunos aspectos que considero de interés:

El primero va en relación a destacar la capacidad e intención de calar en las estructuras institucionales e incidir desde las prácticas cotidianas en las formas de dar asistencia. Ello implica una posición activa, de transformación, en oposición a la de reproducción de esquemas y sistemas de valores preestablecidos.

El segundo se anuda a la vinculación del concepto de salud al de ciudadanía, a través de la idea de salud como concepto integral, derecho básico y producto social. La presencia de intervenciones terapéuticas, que tienden a jerarquizar las propuestas comunitarias, la 
solidaridad social y la recuperación de la historicidad dan cuenta de ello.

El tercer aspecto, trae a la discusión el concepto de trabajo, que es considerado como productor de valores, poderes y posibilidades de ampliar y fortalecer las relaciones sociales. Al proponer, estas modalidades y dispositivos, la generación de espacios laborales en los cuales el trabajo se constituye como eje organizador, se lo considera como un medio para lograr espacios más inclusivos e integradores a través de la recuperación de las tramas sociales, especialmente las más cercanas (personales, familiares y comunitarias).

Por lo tanto, se plantean acciones innovadoras tanto en el seno del campo de la salud mental como en el campo de lo social, debido a que proponen nuevas formas de organización laborales más beneficiosas y adecuadas para las personas en situación de vulnerabilidad psico social.

El último aspecto, y no por ello menos importante, está dado en que las experiencias estudiadas tienen al ámbito público como espacio de acción. Ello habla de la posibilidad de incidencia desde "abajo hacia arriba" en las políticas públicas sanitarias, que a través de experiencias pequeñas o acotadas -en número- demuestran la efectividad o el alcance de nuevas modalidades.

Aunque este trabajo significa una primera aproximación a las prácticas de algunos terapistas ocupacionales, acotadas a una realidad que hace foco en determinados dispositivos de atención, es su pretensión destacar la importancia del papel de la Terapia Ocupacional frente a los desafíos que plantea la asistencia en salud mental.

La actuación del terapista ocupacional como transformador social, la vinculación salud/ ciudadanía, el trabajo como articulador social y la incidencia en las políticas públicas son las tramas sobre los cuales informa el presente trabajo, aspirando ser aporte para futuras proyecciones de estos temas.

\section{Agradecimientos}

Especial agradecimiento a las colegas terapistas ocupacionales entrevistadas, que hicieron posible este estudio a través de la generosa concesión de su tiempo y experiencia.

\section{ReferenCias Bibliográficas}

(1) RUBINSTEIN, S. Microempredimiento productivo en Tercera Edad, en libro Terapia Ocupacional. Trabajo y Comunidad. Serie 3, Buenos Aires, Ed. COLTOA, 1999; p. 93.

(2) CLÉRICl, E, ISLA, E. Microemprendimiento con hemipléjicos adultos, en libro Terapia Ocupacional. Trabajo y Comunidad. Serie 3, Buenos Aires, Ed. COLTOA, 1999; p. 101.

(3) NICASIO, F. Proyectos de inserción en el trabajo: producción de autonomía, itinerarios de ciudadanía, en Revista Materia Prima, Año3, № 11, 1997, p. 23.

(4) PAGANIZZI, L. Actividad. Lenguaje particular, Bs. As, Edigraf, 1997.

Actuaçao da terapia ocupacional no corpo marcado" VII Congreso Brasileiro de Terapia Ocupacional Octubre de 2001, [consulta: mayo 2006] disponible en URL www.terapia-ocupacional.com, Inserción social y trabajo informal. Experiencia y resultados. Avatares de la crisis, 2005. [consulta: mayo 2006] disponible en URL www.terapia-ocupacional.com

(5) MARTíNEZ, R. Análisis funcional y análisis ocupacional. Apuntes de Cátedra, Teoría y Técnica de Terapia ocupacional I. Carrera de Terapia ocupacional. Universidad de Buenos Aires, 1997. La Actividad y la Ocupación, publicado en Revista Terapia ocupacional Materia Prima, $n^{\circ} 14,2000$, p. 9.

La Terapia Ocupacional. Historia y actualidad. Apuntes de Cátedra, Materia: Teoría y Técnica de Terapia ocupacional, Carrera de Terapia ocupacional, Universidad de Buenos Aires, 1998.

Teóricos XI y XII: La intervención, Materia: Teoría y Técnica de Terapia ocupacional I. Carrera de Terapia Ocupacional. Universidad de Buenos Aires, 1997. 
(6) SCHIAPPA, J. La leyenda continúa. Temas y narraciones en Salud Mental. Modalidad rionegrina, Publifadecs- Universidad Nacional del Comahue Río Negro, Argentina, 2003; cap. 3, p. 37; cap. 8, p. 139.

(7) SARACENO, B. Libertando identidades. Da reabilitacao psicossocial â cidadania possível. Primera Edición, Brasil, Instituto Franco Basaglia, Tê Corá Editora, 1999.

A concepcao de rehabilitacao psicossocial como referencia para as intervencoes terapeuticas em saude mental, en revista de Terapia Ocupacional de la Universidad de San Pablo, v. 9/1, 1998, p. 26:31.

(8) ANDER, E. Manual de metodología de la investigación, El Cid Editor, 1983.

(9) SOUZA, M. El desafío del conocimiento. Investigación cualitativa en Salud, Editorial Lugar, Buenos Aires, 1997; p. 175.

(10) ROGER , E. Complejidad, cultura y solidaridad, en apuntes de clase 1-Cátedra Abierta de Solidaridad, Prof Oscar Garcia, Universidad Nacional de San Martín, 2005, p. 30.

(11) CASTEL, R. La metamorfosis de la cuestión social. Primera Edición, Buenos Aires, Editorial Paidós, 1997. 